\title{
Mid-upper arm circumference $v$. weight-for-height $Z$-score for predicting mortality in hospitalized children under 5 years of age
}

\author{
Sakshi Sachdeva ${ }^{1}$, Pooja Dewan ${ }^{1}$, Dheeraj Shah ${ }^{1}$, Rajeev Kumar Malhotra ${ }^{2}$ \\ and Piyush Gupta ${ }^{1, *}$ \\ 'Department of Pediatrics, University College of Medical Sciences, Dilshad Garden, New Delhi 110095 , \\ India: ${ }^{2}$ Department of Biostatistics, University College of Medical Sciences, Delhi, India
}

Submitted 17 April 2015: Final revision received 30 January 2016: Accepted 7 March 2016: First published online 6 April 2016

\begin{abstract}
Objective: To compare the performance of mid-upper arm circumference (MUAC) against weight-for-height $Z$-score (WHZ) for predicting inpatient deaths in children under 5 years of age.

Design: Diagnostic test accuracy study.

Setting: Paediatric emergency department of a tertiary care hospital catering to semi-urban and rural population in Delhi, India.

Subjects: Hospitalized children ( $n$ 1663) aged 6 months to 5 years, for whom discharge outcome was available, were consecutively recruited over 14 months. MUAC $(\mathrm{cm})$, weight $(\mathrm{kg})$ height $(\mathrm{cm})$, clinical details and the outcome were recorded. MUAC (index test) was compared with WHZ based on the WHO growth standards (reference test) for predicting the outcome.

Results: One hundred and twenty-four $(7 \%)$ children died during hospital stay. Both MUAC $<11.5 \mathrm{~cm}$ (adjusted OR (95\% CI): 3.7 (2.43, 5.60), $P<0.001)$ and WHZ $<-3$ (2.0 (1.37, 2.99), $P<0.001)$ served as independent predictors of inpatient mortality. However, MUAC was a significantly better predictor of mortality compared with WHZ in terms of area under the receiver-operating characteristic curve (MUAC $=0.698$, WHZ $=0.541, P<0.001)$. MUAC $<11.5 \mathrm{~cm}$ had the best trade-off of sensitivity and specificity for predicting inpatient mortality. A combination of $\mathrm{WHZ}<-3$ and/or MUAC $<11.5 \mathrm{~cm}$ did not significantly improve the predictive value over that of MUAC/ WHZ, assessed individually.

Conclusion: MUAC $<11.5 \mathrm{~cm}$ is a better predictor of mortality in hospitalized under-5 children, as compared with $\mathrm{WHZ}<-3$. It should be measured in all emergency settings to identify the children at higher risk of death.
\end{abstract}

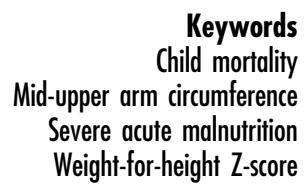

Keywords

Child mortality Severe acute malnutrition Weight-for-height Z-score
Directly or indirectly, malnutrition contributes to nearly two-thirds of global mortality caused by pneumonia, diarrhoea, measles and other infections among children under 5 years of age (under- $5 \mathrm{~s})^{(1,2)}$. In hospitalized Indian children, malnutrition has been shown to increase the risk of mortality up to six times in diarrhoea ${ }^{(3-5)}$ and up to six times in acute respiratory tract infections ${ }^{(6,7)}$. To achieve the UN Millennium Development Goal 4, which aims to further reduce under-5 mortality ${ }^{(8)}$, it is imperative to curtail child deaths occurring as a consequence of malnutrition. The first step in this direction will be to identify and manage the set of malnourished children at risk for imminent death.

The WHO has defined severe acute malnutrition (SAM) in 6-59-month-old children as a weight-for-height $<-3 \mathrm{SD}$ (severe wasting) of the reference population ${ }^{(9)}$. The selection of weight-for-height $Z$-score (WHZ) over other anthropometric criteria, namely weight-for-age $Z$-score (WAZ) or height-for-age $Z$-score (HAZ), is based on the fact that it has been shown to be an indicator of not only present nutritional status but also involves measurement of height that can be utilized to assess past nutritional status. However, the use of WHZ for identification of SAM is associated with some inherent pitfalls, especially in the emergency setting. Difficulties arise owing to the inability to accurately weigh or measure length in sick children; the non-availability of calibrated weighing scales and height boards; the need for reference charts at all times; and the complex calculations to derive and interpret WHZ. Further, WHZ is a statistically derived parameter which depends on the nutritional status of the population from which the $Z$-score has been derived. Recognizing these operational difficulties, in 2009 the UN endorsed mid-upper arm circumference (MUAC) $<11.5 \mathrm{~cm}$ to be an 
age- and sex-independent diagnostic criterion for SAM, alongside $\mathrm{WHZ}<-3^{(9)}$. However, MUAC-based and WHZbased malnutrition diagnoses have shown poor correlation and the children identified as SAM based on one criterion are often missed if diagnosed using the other $^{(10,11)}$. Therefore, the question arises of the choice of MUAC $v$. WHZ as the criterion for selecting hospital admission among severely malnourished children in resource-poor countries.

Several studies have established the superiority of MUAC over other anthropometric indices like weight-for-age, heightfor-age, weight-for-height, WAZ and HAZ as a criterion to predict mortality among under-5 African children in the community ${ }^{(12-18)}$ as well as among hospitalized African children ${ }^{(10,19)}$. A few similar studies have also been done among Asian children in the community ${ }^{(20-22)}$. However, very few studies have directly compared MUAC $<11.5 \mathrm{~cm}$ with $\mathrm{WHZ}<-3$ for predicting child mortality ${ }^{(10,18)}$. As the body composition and aetiology and type of SAM (kwashiorkor or marasmus) is likely to be different in Asian children, it is pertinent to validate these results in these settings ${ }^{(23,24)}$. Moreover, results from community-based studies should not be extrapolated to hospitalized children as the disease-related morbidity amplifies the effects of undernutrition in them. Following admission some children lose weight, consequently increasing the risk of nutritional deterioration.

We conducted the present study to determine the performance of MUAC compared with WHZ for predicting deaths among hospitalized children aged 6 months to 5 years in Indian settings. We also aimed to determine the best cut-off value of MUAC to predict mortality in these children.

\section{Methods}

The study was conducted in the Paediatric Emergency Department of Guru Tegh Bahadur Hospital, a tertiary hospital catering predominantly to the urban poor population of East Delhi and the rural population of western Uttar Pradesh in northern India.

\section{Enrolment}

All consecutive children, aged 6 months to 5 years, admitted to the paediatric emergency department during the study period from February 2012 to March 2013, were considered eligible for recruitment. Children with obesity, congenital anomalies, gross ascites, generalized anasarca, non-nutritional short stature, admitted for diagnostic purpose and for whom outcome on discharge could not be recorded (left against medical advice, referred to other hospitals) were excluded after thorough clinical examination.

\section{Assessment}

Data were collected in a cross-sectional manner on recruitment by the first author (S.S.), who obtained the clinical and sociodemographic details and recorded anthropometric parameters. MUAC was measured with a non-stretchable Teflon measuring tape (Seca, Germany; sensitivity $1 \mathrm{~mm}$ ), midway between the acromion and the tip of the olecranon process marked with elbow bent at $90^{\circ}$. MUAC was taken with the child's arm hanging freely by the side with elbow extended and palm towards the thigh, and the tape encircled around the arm at the marked midpoint, so that it lay flat around the arm without compressing the skin or underlying tissue ${ }^{(25)}$.

Children were weighed naked or with a bare minimum of clothes and without shoes, with the weighing scale kept on a firm even surface and zero checked and tared before each weighing. Infants were weighed on a pan type of digital weighing scale (MI Goldtech, Merino International, India; sensitivity $5 \mathrm{~g}$ ) and older children who could stand by themselves were weighed on a platform-type weighing scale (Merino International; sensitivity $10 \mathrm{~g}$ ) ensuring that the child stood in the centre of the platform without holding on to anything ${ }^{(25)}$.

Length was measured in children less than 2 years old using an infantometer, with the child placed straight and supine on the horizontal board and the Frankfurt plane of the head kept perpendicular to the horizontal with the help of an assistant; length was recorded between the headpiece and the footpiece. Standing height was measured for children aged more than 2 years on the stadiometer (Merino International; sensitivity $1 \mathrm{~mm}$ ) with the child standing barefoot on the platform and ensuring that the back of the head, shoulder blades, buttocks, calves and heels were touching the vertical board. The child was asked to look straight ahead with the Frankfurt plane horizontal ${ }^{(25)}$.

WHZ was derived using the WHO Anthro software ${ }^{(26)}$. This software uses WHO reference standards for growth in under-5 children ${ }^{(27)}$. Underweight and severe underweight were defined as WAZ $<-2$ and $\mathrm{WAZ}<-3$, respectively. Stunting and severe stunting were defined as HAZ $<-2$ and $\mathrm{HAZ}<-3$, respectively. Wasting and severe wasting were defined as $\mathrm{WHZ}<-2$ and $\mathrm{WHZ}<-3$, respectively ${ }^{(28,29)}$. All recruited children were treated according to the standard protocols (as per hospital policy), depending on the child's primary diagnosis. Children identified with SAM were treated with broad-spectrum antibiotics, nutrient supplements, F75 and F100 therapeutic diets, according to the WHO guidelines ${ }^{(29)}$ as modified by the Indian Academy of Pediatrics ${ }^{(30)}$.

Outcome was recorded as live discharge or death.

\section{Sample size}

We planned the present study as a diagnostic test accuracy study wherein MUAC was the 'index test' and WHZ was the 'reference test' for predicting inpatient mortality. Expecting the area under the receiver-operating characteristic (ROC) curve as $0.75^{(10)}$ with $10 \%$ relative precision on either side, $80 \%$ power, $\alpha$ error $=0 \cdot 05$, and considering mortality of 
$8 \%$ in children aged 6 months to 5 years (based on our previous hospital records), we planned to enrol patients until 124 deaths were attained.

\section{Statistical methods}

Baseline characteristics of the study population were computed as proportions for categorical variables, and as mean and standard deviation or median and interquartile range (IQR) for continuous variables. Normality of $Z$-scores and continuous variables was tested with the Shapiro-Wilks method and none of the continuous variables fulfilled the normality condition. These characteristics were further compared between children with different outcomes (survived/died) by the Mann-Whitney $U$ test. ROC curves were constructed to ascertain the predictive values of MUAC, WHZ, WAZ and HAZ for inpatient mortality in terms of area under the curve (AUC) with 95\% confidence interval. Areas under ROC curves were compared using the $\chi^{2}$ test and multiple pairwise comparisons were done, with $P<0 \cdot 01$ considered significant as per Bonferroni adjustment. The data were analysed using the statistical software package IBM SPSS Statistics Version 20 and AUC values were compared using Medcalc trial version 14.12.0. The sensitivity, specificity, positive predictive value and negative predictive value with $95 \%$ confidence intervals for inpatient mortality at different MUAC cut-off values (MUAC $<10.5 \mathrm{~cm},<11 \mathrm{~cm}$, $<11.5 \mathrm{~cm},<12 \mathrm{~cm}$ and $<12.5 \mathrm{~cm}$ ), WHZ $<-3$, WAZ $<-3$, $\mathrm{HAZ}<-3$, and various combinations of these anthropometric parameters, were calculated. The Kruskal-Wallis test was used to compare the characteristics across the groups and the Mann-Whitney $U$ test with Bonferroni correction was applied for multiple corrections. Since the anthropometric indicators MUAC, WHZ, WAZ, and HAZ are collinear, multivariable logistic regression models were fitted considering mortality as the dependent variable. Odds ratios of mortality (adjusted for age and sex) were calculated for $\mathrm{HAZ}<-3$, WAZ $<-3, \mathrm{WHZ}<-3$ and $\mathrm{MUAC}<11.5 \mathrm{~cm}$.

\section{Results}

Of 2389 children (aged 6 months to 5 years) hospitalized during the study period, 1959 (82.0\%) were eligible for inclusion. Figure 1 depicts a flowchart depicting recruitment of children for the study.

\section{Baseline characteristics}

Of the 1663 children finally included, $24.5 \%$ ( $n$ 407) were infants (aged 6-11 months). The median age of the study population was 23 (IQR 12-37) months. Seizure disorder ( $n$ 533, 32.1\%), pneumonia ( $n$ 323, 19.4\%) and diarrhoea ( $n$ 206, 12.4\%) were the most common diagnoses at admission; besides nutritional anaemia ( $n$ 77, 4.6\%), meningoencephalitis ( $n$ 106, 6.4\%), poisoning ( $n 92,5.5 \%$ ), septicaemia ( $n$ 48, 2.9\%), infections (malaria and dengue

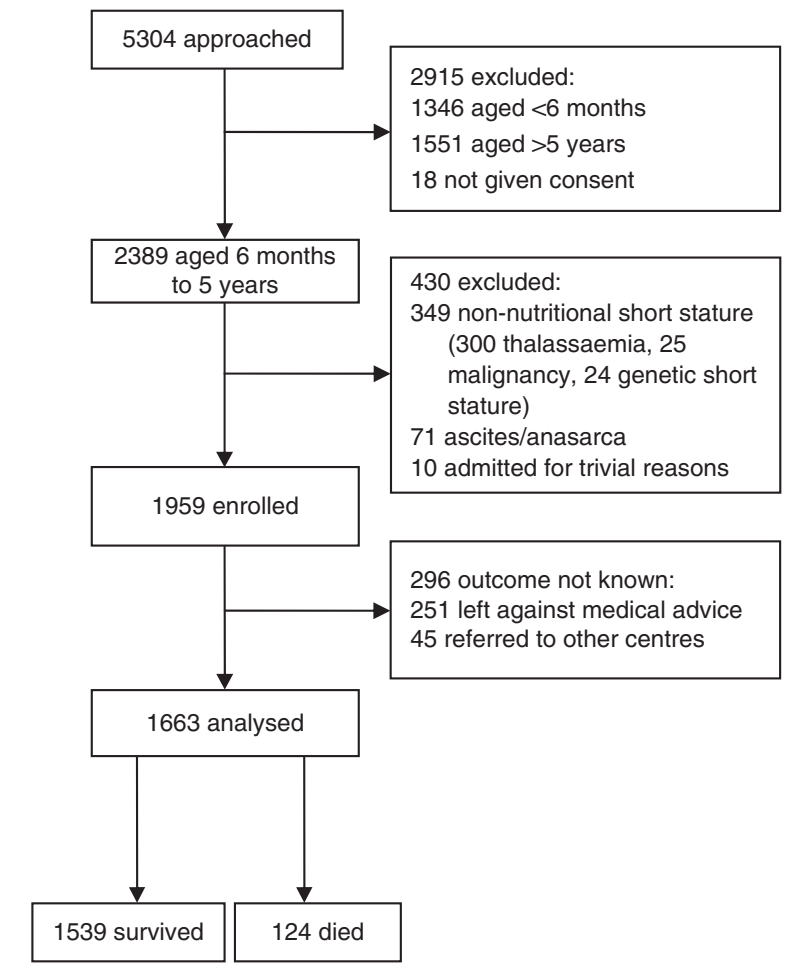

Fig. 1 Flowchart depicting the stepwise recruitment of participants for assessing the predictive value of mid-upper arm circumference $v$. weight-for-height Z-score for inpatient mortality in hospitalized children under 5 years of age, Delhi, India, February 2012-March 2013

fever ( $n 26$ each, 1.6\% each), enteric fever ( $n$ 25, 1.5\%), tuberculosis ( $n$ 26, 1.6\%)), bronchial asthma ( $n$ 18, 1.1\%), acute flaccid paralysis ( $n$ 10, $0.6 \%$ ) and other illnesses (congenital heart disease, tonsillitis, liver abscess, urinary tract infections, hepatitis and glomerulonephritis ( $n$ 147, $8.8 \%)$ ). Major clinical problems at admission included one or more of the following: fever ( $n$ 1079, 64.9\%), convulsions ( $n$ 673, 40.5\%), fast breathing ( $n$ 442, 26.6\%), altered sensorium ( $n$ 118, 7.1\%) and dehydration ( $n$ 241, 14.5\%). About $34 \%$ ( $n$ 562) of children were unimmunized or partially immunized. The median duration of presenting illness was 3 (IQR 1-7) d. Thirty-six per cent of children (607/1663) had clinical features of vitamin deficiency at admission; and $77.4 \%(868 / 1121)$ had laboratory evidence of anaemia $(\mathrm{Hb}<11 \mathrm{~g} / \mathrm{dl})$, of these $20.4 \%$ ( $n$ 177) had severe anaemia $(\mathrm{Hb}<7 \mathrm{~g} / \mathrm{dl})$.

\section{Anthropometric profile at admission}

Mean WHZ, WAZ and HAZ for the enrolled children were -1.5 (SD 2.0), $-2 \cdot 2$ (SD 1.6) and -1.9 (SD 1.7), respectively. Overall, 849 (51.1\%) children were underweight, 788 $(47.4 \%)$ were stunted and $643(38.7 \%)$ children were wasted. Severe underweight, severe stunting and severe wasting were present in 482 (29.0\%), 420 (25.3\%) and 372 (22.4\%) children, respectively. The median MUAC was 
$13 \cdot 0$ (IQR 12.2-14.0) $\mathrm{cm}$. MUAC $<11.5 \mathrm{~cm}$ was seen in 219 (13.2\%) children; of these, seventy-five children had MUAC $<11.5 \mathrm{~cm}$ but WHZ $\geq-3$, while 144 children had both $\mathrm{WHZ}<-3$ and MUAC $<11.5 \mathrm{~cm}$. Overall, WHZ $<-3$ was seen in 372 children (228 children had MUAC $\geq 11.5 \mathrm{~cm}$ but $\mathrm{WHZ}<-3$; Fig. 2). Only seventeen children had oedematous malnutrition.

\section{Outcome}

Overall, 124 children (7.5\%) died; the remaining 1539 (92.5\%) improved and were discharged. Mortality was comparable in infants $(37 / 407,9 \cdot 1 \%)$ and older children (87/1256, 6.9\%; $P=0.15)$. Children who died had significantly lower values for MUAC, head circumference, BMI, WAZ and HAZ (Table 1). The mortality percentages for MUAC $<11.5 \mathrm{~cm}$, severe wasting and severe stunting were $18.7 \%$ (41/219), $11 \cdot 8 \%$ (44/372) and 8.1\% (34/420), respectively. Of children having both MUAC $<11.5 \mathrm{~cm}$ and WHZ $<-3,18 \cdot 1 \%(26 / 144)$ died (Table 2). The distribution of deaths in various categories of undernutrition is shown in Table 3.

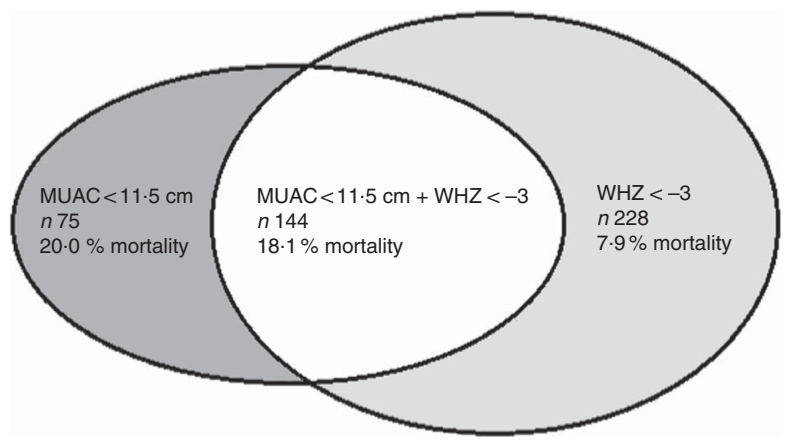

Fig. 2 The sets of hospitalized children under 5 years of age with mid-upper arm circumference (MUAC) $<11.5 \mathrm{~cm}$ and weight-for-height Z-score $(\mathrm{WHZ})<-3$, individually and in combination, Delhi, India, February 2012-March 2013

\section{Differences in children identified by mid-upper arm circumference and weight-for-beight Z-score}

Although the sensitivity and specificity of MUAC $<11.5 \mathrm{~cm}$ and $\mathrm{WHZ}<-3$ for children at risk of dying appeared roughly similar, the sets of children identified by these nutritional indices differed, with only partial overlap (Table 2, Fig. 2). The mortality rate was significantly higher among children with MUAC $<11.5 \mathrm{~cm}(18.7 \%)$ as compared with those with $\mathrm{WHZ}<-3(11 \cdot 8 \%)$. Mortality was lower in children with $\mathrm{WHZ}<-3$ and MUAC $>11.5 \mathrm{~cm}$ (7.9\%; Fig. 2).

\section{Prediction of inpatient mortality}

ROC curve analysis for MUAC, WHZ, WAZ and HAZ showed MUAC as a significantly better predictor $(P<0.001)$ of inpatient mortality, with AUC $=0.698(95 \%$ CI $0.650,0.746)$, compared with $\mathrm{AUC}=0.541$ (95\% CI $0.480,0 \cdot 602)$ for $\mathrm{WHZ}, \mathrm{AUC}=0.574(95 \%$ CI $0.520,0.629)$ for $\mathrm{WAZ}$ and $\mathrm{AUC}=0.562$ (95\% CI $0.513,0.611)$ for HAZ (Fig. 3). There was no significant difference among the AUC of WHZ, WAZ, and HAZ ( $P$ value: HAZ $v$. WAZ $=$ $0 \cdot 68$, HAZ $v . \mathrm{WHZ}=0 \cdot 60, \mathrm{WAZ} v$. WHZ $=0 \cdot 04)$. The sensitivity and specificity of various anthropometric indicators including $\mathrm{MUAC}<11.5 \mathrm{~cm}$ and $\mathrm{WHZ}<-3$ for predicting inpatient mortality are shown in Table 4. WHZ $<-3$ was a better predictor of mortality in younger children (aged 6-11 months) compared with MUAC $<11.5 \mathrm{~cm}$, which performed better in older children (aged 12-60 months; data not shown).

A multivariable logistic regression model adjusted for age, and sex showed highest association of mortality with MUAC $<11.5 \mathrm{~cm}$ (adjusted OR =3.689; 95\% CI 2.430, 5.600; $P<0.001$ ) followed by $\mathrm{WHZ}<-3$ (adjusted $\mathrm{OR}=2 \cdot 025$ $95 \%$ CI $1.373,2.987 ; P<0.001)$ and WAZ $<-3$ (adjusted $\mathrm{OR}=1 \cdot 602 ; 95 \%$ CI $1 \cdot 096,2 \cdot 340 ; P=0 \cdot 015)$. HAZ $<-3$ (adjusted $\mathrm{OR}=1 \cdot 179 ; 95 \%$ CI $0.779, \quad 1.784 ; \quad P=0.435$ )

Table 1 Comparative characteristics of children who died $v$. survivors among hospitalized children under 5 years of age, Delhi, India, February 2012-March 2013

\begin{tabular}{|c|c|c|c|c|c|}
\hline & \multicolumn{2}{|c|}{ Died (n 124) } & \multicolumn{2}{|c|}{ Survived ( $n$ 1539) } & \multirow[b]{2}{*}{$P$ value* } \\
\hline & Median & IQR & Median & IQR & \\
\hline Age (months) & 18 & $11-37$ & 23 & $12-37$ & 0.130 \\
\hline Weight $(\mathrm{kg})$ & $8 \cdot 15$ & $6 \cdot 2-11 \cdot 3$ & 9.1 & $7 \cdot 2-11.4$ & 0.009 \\
\hline Length or height $(\mathrm{cm})$ & $75 \cdot 1$ & $69 \cdot 0-87 \cdot 0$ & 79.9 & $70 \cdot 3-88.5$ & 0.018 \\
\hline Mid-upper arm circumference $(\mathrm{cm})$ & $12 \cdot 1$ & $11 \cdot 2-13 \cdot 1$ & $13 \cdot 1$ & $12 \cdot 3-14 \cdot 1$ & $<0.001$ \\
\hline Head circumference $(\mathrm{cm})$ & $44 \cdot 2$ & $42 \cdot 0-46 \cdot 8$ & $45 \cdot 1$ & $42 \cdot 6-47 \cdot 1$ & 0.010 \\
\hline $\mathrm{BMI}\left(\mathrm{kg} / \mathrm{m}^{2}\right)$ & 13.6 & $11 \cdot 9-16 \cdot 4$ & 14.5 & $12 \cdot 9-15.9$ & 0.047 \\
\hline Weight-for-age Z-score & $-2 \cdot 34$ & $-4.04-1.27$ & -2.02 & $-3 \cdot 17-1.01$ & 0.006 \\
\hline Height-for-age $Z$-score & $-2 \cdot 22$ & $-3.20-1.48$ & -1.90 & $-3.01-0.77$ & 0.021 \\
\hline Weight-for-height $Z$-score & -1.98 & $-3 \cdot 70-0.18$ & -1.46 & $-2 \cdot 75-0.24$ & 0.130 \\
\hline BMI Z-score & -2.01 & $-3.79-0.43$ & -1.34 & $-2.68-0.09$ & 0.034 \\
\hline Duration of presenting illness $(d)$ & 4 & $2-8$ & 2 & $1-7$ & $<0.001$ \\
\hline $\mathrm{Hb}(\mathrm{g} / \mathrm{dl})$ & 9.2 & $7 \cdot 3-11 \cdot 0$ & 9.5 & $8 \cdot 1-10 \cdot 8$ & 0.393 \\
\hline Blood sugar (mg/dl) & 92 & $69-148$ & 99 & $83-120$ & 0.460 \\
\hline
\end{tabular}

IQR, interquartile range.

${ }^{*}$ Compared using the Mann-Whitney $U$ test. 
Table 2 Characteristics of children admitted with/without mid-upper arm circumference (MUAC) $<11.5 \mathrm{~cm}$ and/or weight-for-height $Z$-score $(\mathrm{WHZ})<-3$ among hospitalized children under 5 years of age, Delhi, India, February 2012-March 2013

\begin{tabular}{|c|c|c|c|c|c|c|c|c|}
\hline \multirow[b]{2}{*}{ Clinical feature } & \multicolumn{2}{|c|}{$\begin{array}{c}\text { MUAC } \geq 11.5 \mathrm{~cm} \text { and } \\
W H Z \geq-3(n 1216)\end{array}$} & \multicolumn{2}{|c|}{$\begin{array}{c}M U A C<11.5 \mathrm{~cm} \text { and } \\
W H Z \geq-3(n 75)\end{array}$} & \multicolumn{2}{|c|}{$\begin{array}{c}\mathrm{MUAC}<11.5 \mathrm{~cm} \text { and } \\
\mathrm{WHZ}<-3(n 144)\end{array}$} & \multicolumn{2}{|c|}{$\begin{array}{c}\text { MUAC } \geq 11.5 \mathrm{~cm} \text { and } \\
W H Z<-3(n 228)\end{array}$} \\
\hline & $n$ or Median & $\%$ or IQR & $n$ or Median & $\%$ or IQR & $n$ or Median & $\%$ or IQR & $n$ or Median & $\%$ or IQP \\
\hline Age $<1$ year, $n$ and $\%$ & 267 & $22 \cdot 0$ & 38 & $50 \cdot 7$ & 59 & $41.0 \%$ & 43 & $18 \cdot 8$ \\
\hline Age (months), median and IQR & 24 & $12-40$ & 11 & $9-24$ & 13 & $9-23$ & 24 & $13-36$ \\
\hline Duration of illness (d), median and IQR & 2 & $1-5$ & 5 & $3-11.75$ & 7 & $2-18$ & 3 & $1-7$ \\
\hline Seizure, $n$ and $\%$ & 539 & $44 \cdot 3$ & 17 & 22.4 & 20 & 13.9 & 97 & $42 \cdot 5$ \\
\hline Fast breathing, $n$ and $\%$ & 303 & 24.9 & 31 & $41 \cdot 3$ & 45 & $31 \cdot 2$ & 63 & $27 \cdot 6$ \\
\hline Diarrhoea, $n$ and \% & 179 & 14.7 & 20 & $26 \cdot 7$ & 75 & $52 \cdot \overline{1}$ & 51 & $22 \cdot 4$ \\
\hline Dehydration, $n$ and $\%$ & 122 & $10 \cdot 0$ & 14 & $18 \cdot 7$ & 65 & $45 \cdot 1$ & 40 & 17.5 \\
\hline $\mathrm{Hb}(\mathrm{g} / \mathrm{dl})$, median and IQR & $9 \cdot 7$ & $8 \cdot 2-10.9$ & 8.5 & $5 \cdot 9-10 \cdot 2$ & $9 \cdot 1$ & $7 \cdot 0-10.5$ & 9.5 & $8 \cdot 1-10 \cdot 8$ \\
\hline Blood sugar (mg/dl), median and IQR & 100 & $85-124$ & 103 & $80-143$ & 90 & $74-109$ & 99 & $82-125$ \\
\hline Duration of stay $(\mathrm{h})$, median and IQR & 62 & $26-114$ & 96.5 & $44-190$ & 121 & 49-197 & 67 & $29-137$ \\
\hline Outcome (mortality), $n$ and $\%$ & 65 & $5 \cdot 3$ & 15 & 20.0 & 26 & $18 \cdot 1$ & 18 & 7.9 \\
\hline
\end{tabular}

IQR, interquartile range.

Table 3 Number of admissions and inpatient deaths, by nutritional indices, among hospitalized children under 5 years of age, Delhi, India, February 2012-March 2013

\begin{tabular}{|c|c|c|c|c|c|c|c|c|c|c|c|c|c|}
\hline \multirow[b]{3}{*}{$Z$-score } & \multicolumn{9}{|c|}{ Nutritional indices reported as $Z$-scores } & & & & \\
\hline & \multicolumn{3}{|c|}{ Weight-for-height $Z$-score } & \multicolumn{3}{|c|}{ Weight-for-age $Z$-score } & \multicolumn{3}{|c|}{ Height-for-age Z-score } & \multicolumn{4}{|c|}{ Mid-upper arm circumference $(\mathrm{cm})$} \\
\hline & $n$ & Died & $\%$ & $n$ & Died & $\%$ & $n$ & Died & $\%$ & & $n$ & Died & $\%$ \\
\hline$<-4$ & 194 & 25 & $12 \cdot 9$ & 245 & 31 & $12 \cdot 6$ & 180 & 13 & $7 \cdot 2$ & $<11 \mathrm{~cm}$ & 127 & 25 & $19 \cdot 7$ \\
\hline$<-3$ & 372 & 44 & 11.8 & 482 & 48 & 9.9 & 420 & 34 & 8.1 & $<11.5 \mathrm{~cm}$ & 219 & 41 & $18 \cdot 7$ \\
\hline$<-2$ & 643 & 61 & 9.5 & 849 & 73 & 8.6 & 788 & 71 & $9 \cdot 0$ & $<12 \mathrm{~cm}$ & 298 & 50 & $16 \cdot 8$ \\
\hline$<-1$ & 973 & 77 & 7.9 & 1260 & 99 & $7 \cdot 8$ & 1181 & 98 & 8.3 & $<12.5 \mathrm{~cm}$ & 512 & 73 & $14 \cdot 2$ \\
\hline$\geq-1$ & 690 & 47 & $6 \cdot 8$ & 403 & 25 & $6 \cdot 2$ & 482 & 26 & 5.4 & $\geq 12.5 \mathrm{~cm}$ & 1151 & 51 & 4.4 \\
\hline
\end{tabular}

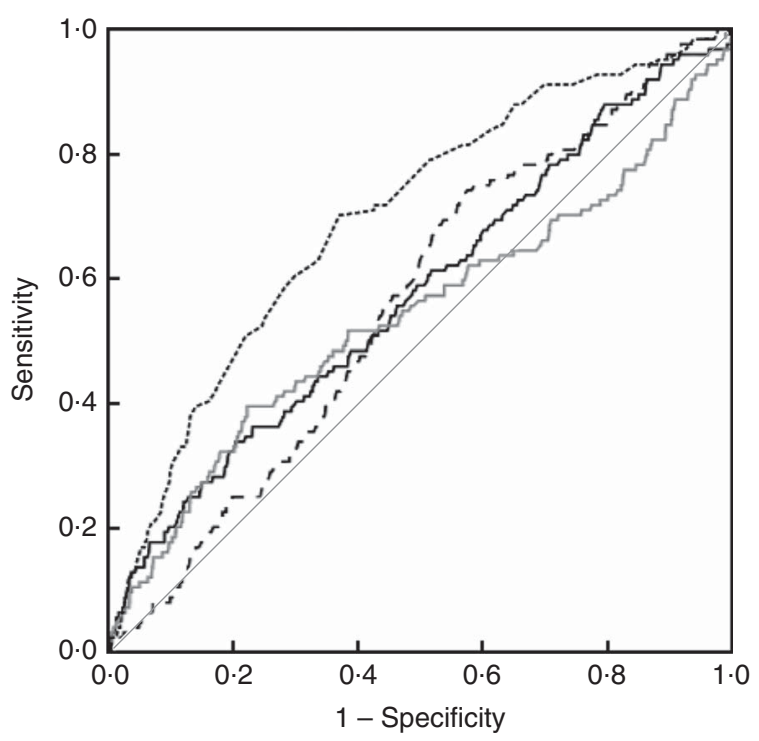

Fig. 3 Receiver-operating characteristic curves showing the ability of mid-upper arm circumference (-- - -), weight-forheight Z-score (-), weight-for-age Z-score (-) and height-for-age Z-score $(---)$, in terms of area under the curve (-represents the line of equality), to predict inpatient mortality in hospitalized children under 5 years of age, Delhi, India, February 2012-March 2013 did not have a significant independent association with inpatient mortality.

Shock $(\mathrm{OR}=20 \cdot 5 ; 95 \% \mathrm{CI} 8 \cdot 2,51 \cdot 2)$, lethargy $(\mathrm{OR}=15 \cdot 3$; $95 \%$ CI 9.9, 23.7), hypoglycaemia (OR $=7 \cdot 7 ; 95 \%$ CI $3 \cdot 0$, 19.8), poor immunization status ( $\mathrm{OR}=3 \cdot 1 ; 95 \% \mathrm{CI} 2 \cdot 1,4 \cdot 5)$, fast breathing $(\mathrm{OR}=2 \cdot 8 ; 95 \% \mathrm{CI} 2 \cdot 0,4 \cdot 1)$, dehydration $(\mathrm{OR}=2 \cdot 3 ; 95 \%$ CI $1 \cdot 5,3 \cdot 5)$, fever $(\mathrm{OR}=1 \cdot 8 ; 95 \%$ CI $1 \cdot 2,2 \cdot 8)$, hyperglycaemia $(\mathrm{OR}=1 \cdot 8 ; 95 \% \mathrm{CI} 1 \cdot 0,3 \cdot 3)$ and diarrhoea $(\mathrm{OR}=1 \cdot 8 ; 95 \% \mathrm{CI} 1 \cdot 2,2 \cdot 7)$ at admission were found to have a significant association with subsequent inpatient mortality.

\section{Discussion}

In our study among hospitalized children aged 6 months to 5 years, we found that MUAC was a significantly better predictor of mortality compared with $\mathrm{WHZ}<-3$, especially in children aged between 1 and 5 years. Out of all anthropometric parameters studied, MUAC cut-off of $<11.5 \mathrm{~cm}$ had the best combination of sensitivity and specificity (sensitivity $33.0 \%$, specificity $88.4 \%$ ) for predicting inpatient mortality. The risk of death in children having $\mathrm{MUAC}<11.5 \mathrm{~cm}$ was more than twice the risk in children having $\mathrm{WHZ}<-3$ and MUAC $>11.5 \mathrm{~cm}$. Combining MUAC $<11.5 \mathrm{~cm}$ with 
Table 4 Performance of anthropometric indicators to predict inpatient mortality among hospitalized children under 5 years of age, Delhi, India, February 2012-March 2013

\begin{tabular}{|c|c|c|c|c|c|c|c|c|}
\hline & \multicolumn{2}{|c|}{ Sensitivity } & \multicolumn{2}{|c|}{ Specificity } & \multicolumn{2}{|c|}{ Positive predictive value } & \multicolumn{2}{|c|}{ Negative predictive value } \\
\hline & $\%$ & $95 \% \mathrm{Cl}$ & $\%$ & $95 \% \mathrm{Cl}$ & $\%$ & $95 \% \mathrm{Cl}$ & $\%$ & $95 \% \mathrm{Cl}$ \\
\hline $\mathrm{WHZ}<-3$ & 35.5 & $27 \cdot 1,44 \cdot 6$ & $78 \cdot 7$ & $76 \cdot 6,80 \cdot 8$ & $11 \cdot 8$ & $8 \cdot 8,15 \cdot 6$ & 93.8 & $92 \cdot 3,95 \cdot 1$ \\
\hline MUAC $<11.5 \mathrm{~cm}$ & $33 \cdot 1$ & $25 \cdot 9,42 \cdot 1$ & 88.4 & $86 \cdot 7,90 \cdot 0$ & $18 \cdot 7$ & $13 \cdot 8,24 \cdot 5$ & 94.2 & $92.9,95.4$ \\
\hline $\mathrm{HAZ}<-3$ & $27 \cdot 4$ & $19 \cdot 7,36 \cdot 1$ & 74.9 & $72 \cdot 7,77 \cdot 1$ & $8 \cdot 1$ & $5 \cdot 7,11 \cdot 1$ & $92 \cdot 7$ & $91 \cdot 2,94 \cdot 1$ \\
\hline$W A Z<-3$ & 38.7 & $30 \cdot 1,49 \cdot 7$ & 71.7 & $69 \cdot 4,74.0$ & 9.9 & $7.4,12 \cdot 9$ & 93.6 & $92 \cdot 0,94.9$ \\
\hline $\mathrm{WHZ}<-3$ and $\mathrm{MUAC}<11.5 \mathrm{~cm}$ & 21.0 & $14 \cdot 2,29 \cdot 2$ & $92 \cdot 4$ & $91 \cdot 0,98 \cdot 7$ & $18 \cdot 2$ & $12 \cdot 2,25 \cdot 5$ & 93.5 & $92 \cdot 2,94 \cdot 7$ \\
\hline $\mathrm{WHZ}<-3$ or $\mathrm{MUAC}<11.5 \mathrm{~cm}$ & 47.5 & $38 \cdot 5,56 \cdot 7$ & 74.8 & $72 \cdot 5,76 \cdot 9$ & $13 \cdot 2$ & $10 \cdot 2,16 \cdot 7$ & 94.6 & $93 \cdot 2,95 \cdot 9$ \\
\hline
\end{tabular}

WHZ, weight-for-height Z-score, MUAC, mid-upper arm circumference; HAZ, height-for-age Z-score; WAZ, weight-for-age Z-score.

WHZ $<-3$ did not significantly improve the diagnostic performance of MUAC $<11.5 \mathrm{~cm}$. WHZ $<-3$ had a comparatively better performance in children younger than 1 year old.

Current diagnostic criteria for SAM in children aged 6 months to 5 years, for admission into a therapeutic feeding programme, include $\mathrm{WHZ}<-3$, or MUAC $<11.5$ $\mathrm{cm}$, or presence of bipedal oedema ${ }^{(31)}$. Several community-based studies have established that MUAC is a convenient and efficacious tool for predicting risk for childhood mortality over other anthropometric measures like weight-for-age $\mathrm{e}^{(12,13,15,16,21)}$, height-for-age $\mathrm{e}^{(12,13,15,16)}$ and weight-for-height ${ }^{(12,13,15,16,20,22)}$ among the under-5 population. Three hospital-based studies ${ }^{(10,19,23)}$ from Africa and one from Asia ${ }^{(32)}$ have also shown that MUAC may be used in preference over WHZ for predicting the risk of death in under- 5 children. A hospital-based study from Kenya ${ }^{(10)}$ validated MUAC $<11.5 \mathrm{~cm}$ to be on a par with $\mathrm{WHZ}<-3$ for identifying hospitalized under-5 children at risk of dying. However, a study from Senegal $^{(18)}$ documented that use of MUAC $<11.5 \mathrm{~cm}$ alone may be better than $\mathrm{WHZ}<-3$ in identifying children at high risk of death, and there was not much additional advantage of using both $\mathrm{WHZ}<-3$ and/or MUAC $<11.5$ $\mathrm{cm}$. Another recent retrospective analysis of 2205 children admitted into a therapeutic feeding programme in Sudan $^{(23)}$ found that children who would have been admitted using MUAC $<11.5 \mathrm{~cm}$ were more severely malnourished, less likely to recover and had higher risk of death than children admitted with $\mathrm{WHZ}<-3$ and MUAC $>$ $11.5 \mathrm{~cm}$. A recent study ${ }^{(32)}$ from India evaluating 6307 children admitted to nutrition rehabilitation centres also documented that MUAC $<11.5 \mathrm{~cm}$ was as effective as $\mathrm{WHZ}<-3$ in identifying children with risk of medical complications or death. We validated these results in an urban poor population in an Indian setting, and documented MUAC $<11.5 \mathrm{~cm}$ to have a better diagnostic performance than $\mathrm{WHZ}<-3$ for identifying hospitalized children aged 6 months to 5 years at risk of dying.

We found that the performance of MUAC $<11.5 \mathrm{~cm}$ was not as good as that of $\mathrm{WHZ}<-3$ for predicting mortality among hospitalized infants. This may be because MUAC is not truly an age-independent measure in infants, who have a higher rate of growth than older children ${ }^{(33)}$. Taking a single cut-off measure of MUAC $<11.5 \mathrm{~cm}$ for all children aged 6 months to 5 years is likely to overestimate malnutrition in younger children as compared with those in the upper range of the age group. Although we found WHZ $<-3$ to be better at predicting mortality in children aged 6-11 months, this has been found questionable elsewhere ${ }^{(34,35)}$ probably due to errors which creep in during the process of converting weight and height into WHZ. Moreover, these studies involved much younger infants aged 0-6 months. This suggests the need for more focused studies to identify anthropometric predictors of short-term mortality in older infants.

Studies in the past have also reported MUAC to be superior in performance to $\mathrm{WHZ}$ in predicting mortality among children. Unlike WHZ, MUAC is less affected by hydration status ${ }^{(36)}$. Sick children often have dehydration at the time of hospitalization due to poor oral intake or due to third-space fluid losses, while a few others may have fluid overload due to congestive heart failure. In such situations MUAC may be a better parameter compared with WHZ to assess nutritional status. The 'muscle mass hypothesis' postulated by Briend and co-workers ${ }^{(37)}$ also states that mortality is directly proportional to the ratio of muscle mass to the mass of energy-requiring organs especially the brain, MUAC being an indirect measure of the muscle mass.

We found that when using MUAC $<11.5 \mathrm{~cm}$ as the sole criterion, we failed to identify one-third of the children at risk for dying. These results are synchronous with some other studies which have shown that MUAC $<11.5 \mathrm{~cm}$ and $\mathrm{WHZ}<-3$ identify different sets of children with partial overlap, and if only one criterion is to be used for identification of children with highest risk of death, it should be MUAC $<11.5 \mathrm{~cm}^{(18,23,32,38)}$.

The strength of our study is that it was carried out in an urban poor population with high background rate of malnutrition and risk of infection. Further, the study was conducted prospectively with anthropometric measurements being taken by a trained resident. The main limitation of our study is that it was carried out in a hospital setting among children who already had medical illnesses at the time of presentation. However, the majority of 
illnesses and deaths in the included children could be categorized as malnutrition-related mortality. Intervention provided during course of the children's stay must have alleviated several complications of malnutrition that could have resulted in death. Also, the children who were discharged from hospital were not followed up for any subsequent mortality. Longitudinal studies in community settings among children identified as severely malnourished by different anthropometric criteria are desirable to conclusively address the issue of the best parameter to identify risk of mortality, but these studies will be difficult to design because of ethical and feasibility issues.

\section{Conclusion}

We conclude that MUAC $<11.5 \mathrm{~cm}$ has a better performance than $\mathrm{WHZ}<-3$ for identifying children at risk of mortality in hospitalized under- 5 children in an urban poor setting, especially in the age group of $1-5$ years. Although $\mathrm{WHZ}<-3$ and MUAC $<11.5 \mathrm{~cm}$ identify different sets of malnourished children, those identified by MUAC have a higher risk of mortality, and thus MUAC $<11.5 \mathrm{~cm}$ alone can be used to triage children for therapeutic interventions in resource-limited settings.

\section{Acknowledgements}

Financial support: This research received no specific grant from any funding agency in the public, commercial or not-for-profit sectors. Conflict of interest: None. Authorship: concept: P.G. and D.S. Study design: P.G., D.S., P.D., S.S. and R.K.M. Literature review: S.S. and P.D. Data collection: S.S. Data analysis: S.S., P.D., R.K.M. and P.G. Interpretation of results: S.S., P.D. and P.G. Drafting the manuscript: S.S., P.D. and R.K.M. Critical inputs on the manuscript: P.G. and D.S. Approved final version: all authors. Ethics of human subject participation: This study was conducted according to the guidelines laid down in the Declaration of Helsinki and all procedures involving human subjects were approved by the ethics committee of the University College of Medical Sciences, Delhi. Written informed consent was obtained from guardians of all children.

\section{References}

1. The Child Health Epidemiology Reference Group (2012) Definitive global childhood causes of death estimates released by CHERG. Not on track to meet Millennium Development Goal 4. http://www.jhsph.edu/departments/ international-health/the-globe/archive/summer2012/childcauses-of-death.html (accessed September 2013).

2. Rice AL, Sacco L, Hyder A et al. (2000) Malnutrition as an underlying cause of childhood deaths associated with infectious diseases in developing countries. Bull World Health Organ 78, 1207-1221.
3. Behera SK, Mohapatra SS, Kar S et al. (1980) Incidence and mortality of hospitalized diarrhea cases. Part III. Indian Pediatr 17, 607-612.

4. Srivastava AK, Bhatnagar JK, Prasad BG et al. (1973) A clinical and aetiological study of diarrhoea in hospitalized children at Lucknow. Indian J Med Res 61, 596-602.

5. Sachdev HP, Kumar S, Singh KK et al. (1991) Risk factors for fatal diarrhea in hospitalized children in India. J Pediatr Gastroenterol Nutr 12, 76-81.

6. Sehgal V, Sethi GR, Sachdev HP et al. (1997) Predictors of mortality in subjects hospitalized with acute lower respiratory tract infections. Indian Pediatr 34, 213-219.

7. Deivanayagam N, Nedunchelian K, Ramasamy S et al. (1992) Risk factors for fatal pneumonia: a case control study. Indian Pediatr 29, 1529-1532.

8. United Nations Development Programme (2006) About MDGs: what they are. http://www.unmillenniumproject. org/goals/index.htm (accessed December 2012).

9. World Health Organization (2009) WHO Child Growth Standards and the Identification of Severe Acute Malnutrition in Infants and Children: A Joint Statement by the World Health Organization and the United Nations Children's Fund. Geneva: WHO.

10. Berkley J, Mwangi I, Griffiths K et al. (2005) Assessment of severe malnutrition among hospitalized children in rural Kenya: comparison of weight for height and mid-upper arm circumference. JAMA 294, 591-597.

11. Fernandez MA, Delchevalerie P \& Van HM (2010) Accuracy of MUAC in the detection of severe wasting with the new WHO growth standards. Pediatrics 126, e195-e201.

12. Vella V, Tomkins A, Ndiku J et al. (1994) Anthropometry as a predictor for mortality among Ugandan children, allowing for socio-economic variables. Eur J Clin Nutr 48, 189-197.

13. Vella V, Tomkins A, Borghesi A et al. (1993) Anthropometry and childhood mortality in northwest and southwest Uganda. Am J Public Health 83, 1616-1618.

14. Yambi O, Latham M, Habicht JP et al. (1991) Nutritional status and the risk of mortality in children 6-36 months old in Tanzania. Food Nutr Bull 13, 271-276.

15. Fawzi WW, Herrera MG, Spiegelman DL et al. (1997) A prospective study of malnutrition in relation to child mortality in the Sudan. Am J Clin Nutr 65, 1062-1069.

16. Vella V, Tomkins A, Nidku J et al. (1992) Determinants of child mortality in south-west Uganda. J Biosoc Sci 24, 103-112.

17. Bern C, Zucker JR, Perkins BA et al. (1997) Assessment of potential indicators for protein-energy malnutrition in the algorithm for integrated management of childhood illness. Bull World Health Organ 75, Suppl. 1, 87-96.

18. Briend A, Maire B, Fontaine O et al. (2012) Mid-upper arm circumference and weight-for-height to identify high-risk malnourished under-five children. Matern Child Nutr 8, 130-133.

19. Dramaix M, Brasseur D, Donnen P et al. (1996) Prognostic indices for mortality of hospitalized children in central Africa. Am J Epidemiol 143, 1235-1243.

20. Alam N, Wojtyniak B \& Rahaman MM (1989) Anthropometric indicators and risk of death. Am J Clin Nutr 49, 884-888.

21. Chen LC, Chowdhury A \& Huffman SL (1980) Anthropometric assessment of energy-protein malnutrition and subsequent risk of mortality among preschool aged children. Am J Clin Nutr 33, 1836-1845.

22. Bairagi R \& Chowdhury MK (1994) Socioeconomic and anthropometric status, and mortality of young children in rural Bangladesh. Int J Epidemiol 23, 1179-1184.

23. Grellety E, Krause LK, Shams Eldin M et al. (2015) Comparison of weight-for-height and mid-upper arm circumference (MUAC) in a therapeutic feeding programme in South Sudan: is MUAC alone a sufficient criterion for admission of children at high risk of mortality? Public Health Nutr 18, 2575-2581. 
24. Ramalingaswami V, Jonsson U \& Rohde J (1996) The Progress of Nations 1996. Nutrition. Commentary: The Asian enigma. http://www.unicef.org/pon96/contents.htm (accessed September 2015).

25. Shah D \& Sachdev HPS (2011) Measuring undernutrition and overnutrition in children. In Public Health Nutrition in Developing Countries. Part I, pp. 108-150 [SC Vir, editor]. New Delhi: Woodhead Publishing India.

26. World Health Organization (2010) Anthro for Personal Computers, Version 3.2.2, 2011: Software for Assessing Growth and Development of the World's Children. Geneva: WHO; available at http://www.who.int/childgrowth/software/en/

27. World Health Organization, Multicentre Growth Reference Study Group (2006) WHO Child Growth Standards: Length/ Height-for-Age, Weight-for-Age, Weight-for-Length, Weightfor-Height and Body Mass Index-for-Age: Methods and Development. Geneva: WHO.

28. Gupta P (2013) Nutrition and health. In Textbook of Pediatrics, 1st ed., pp. 51-83 [P Gupta, editor]. New Delhi: CBS Publishers.

29. World Health Organization (2003) Guidelines for the inpatient treatment of severely malnourished children. http://www. who.int/nutrition/publications/guide_inpatient_text.pdf (accessed March 2016).

30. Bhatnagar S, Lodha R, Choudhury P et al. (2007) IAP guidelines 2006 on hospital based management of severely malnourished children (adapted from the WHO Guidelines). Indian Pediatr 44, 443-461.

31. World Health Organization (2013) Guideline updates on the management of severe acute malnutrition in infants and children. http://www.who.int/nutrition/publications/ guidelines/updates_management_SAM_infantandchildren/en/ (accessed March 2015).

32. Aguayo VM, Aneja S, Badgaiyan N et al. (2015) Mid upperarm circumference is an effective tool to identify infants and young children with severe acute malnutrition in India. Public Health Nutr 18, 3244-3248.

33. World Health Organization (2007) Child Growth Standards: Methods and Development. Head Circumference-for-Age, Arm Circumference-for-Age, Triceps Skinfold-Forage and Subscapular Skinfold-for-Age. Geneva: WHO.

34. Mwangome MK, Fegan G, Fulford T et al. (2012) Mid-upper arm circumference at age of routine infant vaccination to identify infants at elevated risk of death: a retrospective cohort study in the Gambia. Bull World Health Organ 90, 887-894.

35. Mwangome MK \& Berkley JA (2014) The reliability of weight-for-length/height $Z$ scores in children. Matern Child Nutr 10, 474-480.

36. Mwangome MK, Fegan G, Prentice AM et al. (2011) Are diagnostic criteria for acute malnutrition affected by hydration status in hospitalized children? A repeated measures study. Nutr J 10, 92.

37. Briend A, Garenne M, Maire B et al. (1989) Nutritional status, age and survival: the muscle mass hypothesis. Eur J Clin Nutr 43, 715-726.

38. Laillou A, Prak S, de Groot R et al. (2014) Optimal screening of children with acute malnutrition requires a change in current WHO guidelines as MUAC and WHZ identify different patient groups. PLoS One 9, e101159. 